\title{
SOME WEAK EQUIVALENCES FOR CLASSIFYING SPACES
}

\author{
SOLOMON M. JEKEL
}

\begin{abstract}
For topological categories ${ }^{2}$ with fractions we give a model for the loops on the classifying space $\Omega B C^{\prime}$ as a simplicial group which generalizes the fact that $\Omega B E=E$ when $E$ is a topological group. This construction is applied to give some well-known, and some new, examples of weak equivalences between classifying spaces arising in the theory of foliations.
\end{abstract}

Introduction. Let $B \Gamma$ be the classifying space of a topological groupoid $\Gamma$. In [2] the loop space $\Omega B \Gamma$ is described up to weak homotopy as a simplicial group $G_{*} \Gamma$ in a way which generalizes the fact that $\Omega B G=G$ for $G$ a topological group. In this paper we extend the constructions of [2] to include certain topological categories $P$. We prove that when $\varrho$ has a "calculus of fractions" then $\Omega B \circlearrowright=G_{*} \circlearrowright$. The more general construction provides a natural setting in which to obtain weak equivalences between certain classifying spaces arising in the theory of foliations.

The applications are motivated by G. Segal's work on classifying spaces [8]. Segal proves that the classifying space of the discrete monoid of smooth embeddings of $\mathbf{R}^{n}$ into itself is, up to weak equivalence, $B \Gamma_{n}^{\infty}$, the classifying space for codimension- $n$ smooth foliations. We obtain a generalization of Segal's result by carrying out the following program: to compare a monoid $M$ and a groupoid $\Gamma,(1)$ make $M$ into a topological category $\bigodot$ with the same objects as $\Gamma,(2)$ check that $\bigodot$ has fractions, (3) compare $G_{*} e$ with $G_{*} \Gamma$. The result is that this procedure works when $\Gamma$ is both a sheaf and a groupoid over a contractible space, $M$ is the monoid of sections of the source map, and certain simple topological conditions are satisfied.

Peter Greenberg has used Segal's techniques to prove a somewhat different version of our theorem [1].

We conclude with several examples of groupoids and associated monoids (in addition to those of Segal's theorem) which fall into our general framework and arise in the theory of foliations. For example, covered by our result is $\Gamma_{n}^{\omega}$, the germs of real analytic homeomorphisms of $\mathbf{R}^{n}$, and we obtain a weak equivalence of $B \Gamma_{n}^{\omega}$ with both the monoid of real analytic immersions of $\mathbf{R}^{n}$ into itself and with the monoid of real analytic embeddings of $\mathbf{R}^{n}$ into itself.

1. Statement of the main theorem. Let $\Gamma$ be a topological groupoid with contractible object space $\theta$ such that the source and target maps $s, t$ are local homeomorphisms from $\Gamma$ onto $\theta$. For example, $\Gamma$ may be the groupoid with the sheaf topology

Received by the editors April 25, 1983.

1980 Mathematics Subject Classification. Primary 57R32; Secondary 55P10.

${ }^{1}$ This research was partially supported by the National Science Foundation. $0002-9939 / 84 \$ 1.00+\$ .25$ per page 
associated to a pseudogroup of local homeomorphism of $\theta$. Let $s(\Gamma)=\{f: \theta \rightarrow \Gamma \mid$ $s f=$ identity $\}$ be the set of sections of the source. Consider $\varsigma(\Gamma)$ as a discrete monoid under composition induced by $\Gamma$.

Definition. A submonoid $\mathfrak{T}(\Gamma) \subset \delta(\Gamma)$ is called $\Gamma$-complete if

(i) the collection of open sets $\{\operatorname{tm}(\theta)\}, m \in \mathcal{N}(\Gamma)$, forms a basis for the topology of $\theta$, and

(ii) if $U \subset \mathcal{Q}$ is any open set, and $g: U \rightarrow \Gamma$ is a section of the source such that $U \rightarrow \operatorname{tg}(U)$ is a local homeomorphism, then for each $m \in \Re(\Gamma)$ with $\operatorname{tm}(\theta) \subset U$ we must have $g m \in \mathscr{M}(\Gamma)$ :

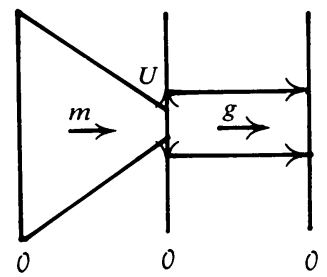

For example $\delta(\Gamma)$ is itself $\Gamma$-complete and $\mathfrak{M}(\Gamma) \subset \delta(\Gamma)$, consisting of all $m \in \delta(\Gamma)$ so that $\theta \rightarrow \operatorname{tm}(\theta)$ is a $1-1$ function, is $\Gamma$-complete.

THEOREM 1. Let $\Gamma$ be a topological groupoid with contractible object space such that the source and target maps are local homeomorphisms. Let $\Re(\Gamma)$ be a $\Gamma$-complete (discrete) monoid. Then $B \mathfrak{T}(\Gamma)$ and $B \Gamma$ have the same weak homotopy type.

The proof of Theorem 1 will be given in $\S 5$. The proof makes use of a generalization of the loop construction of [2] from topological groupoids to topological categories. Before stating this generalization (Theorem 2, §4) we introduce some fundamental constructions for categories.

2. Discrete categories and fractions. Let $\mathcal{C}$ be a discrete small category with objects $\theta$. We identify $e$ with its set of morphisms and $\theta$ with the subset of identity morphisms.

Definition. The reduced set of $C$ is the set $e / \Theta$ obtained by identifying all identity morphisms to a point.

$\circlearrowright / \Theta$ inherits a partial multiplication from $\circlearrowright$ as follows. Let $(x, y) \rightarrow x y$ denote composition in $\mathcal{C}: A \stackrel{y}{\rightarrow} B \stackrel{x}{\rightarrow} C$. Let 1 denote the equivalence class of $\mathcal{C}$ in $e / C$. Define a multiplication $*:(x, y) \rightarrow x * y$ in $\mathrm{C} / \Theta$ by

(i) $x * y=x y$ if $x \neq 1, y \neq 1$ and $x y$ is defined in $\mathrm{C}$,

(ii) $x * 1=1 * x=x$ for $x \in \mathcal{C} / \mathbb{O}$.

Let $N \mathcal{C}$ be the nerve of $\bigodot$. We denote the set of $p$-simplices of $N \mathcal{C}$ by $N_{p} \mathcal{C}$. Let $N \in$ be the subsimplicial set of $N \mathcal{C}$ consisting of identity morphisms so that $N_{p} \theta=$ diagonal $\theta^{p}$. Let $N_{p}(\mathrm{e} / \theta)=N_{p} \mathrm{e} / N_{p}(\vartheta$.

Definition. The nerve of $\mathrm{e} / \Theta$ is the simplicial set $\cup_{p \geqslant 0} N_{p}(\Theta / \Theta)$ with faces and degenericies induced by those of $N \mathrm{C}$.

Definition. The universal group of $e$ is the free group on the elements of $e / \theta$ modulo the relations $x \cdot y=x * y$. Here $x \cdot y$ is the free product, $x * y$ is as above, and a relation holds whenever $x * y$ is defined. We denote this group by $\mathcal{G}_{0} \varrho$. 
Definition. Let $e$ be a discrete category. $e$ is said to have a calculus of right fractions if the following two conditions are satisfied [5].

(1) For each diagram $A \stackrel{x}{\rightarrow} B \stackrel{x}{\leftarrow} C, x, y \in \mathcal{C}$, there is a diagram $A \stackrel{\bar{x}}{\leftarrow} \bar{B} \stackrel{\bar{y}}{\rightarrow} C, \bar{x}$, $\bar{y} \in \mathcal{Q}$ and $x \bar{x}=y \bar{y}$.

(2) If $x, y: A \rightarrow B$, and $z \in \mathcal{C}$ satisfies $z x=z y$, there is a $w \in \mathcal{C}$ so that $x w=y w$.

There is an analogous definition of a calculus of left fractions. We will say that $\mathcal{C}$ has fractions if it has a calculus of left or right fractions.

The following fact is well known; see, for example, [5].

THEOREM A. Let $e$ be a discrete category. If $e$ has fractions then $\mid N \mathcal{C}$, the realization of the nerve of $\mathcal{C}$, is a disjoint union of $K(\pi, 1)$ 's.

The following fact is essential to the sequel and will be proved in $\S 4$.

THEOREM B. Let $巳$ be a discrete category. If $e$ has fractions then $|N(\bigodot / \Theta)|$ is a $K\left(\mathcal{G}_{0} \mathrm{e}, 1\right)$.

3. Topological categories and simplicial fractions. Now let $e$ be a topological category. Let $N \mathcal{C}$ be the simplicial space which is the nerve of $\mathcal{C} . B C$, the classifying space of $\mathrm{C}$, is defined to be $\| N \mathcal{C}_{\|}$.

REMARK ON REALIZATIONS. We will make use of two different realizations of simplicial spaces: $|\cdot|$ and $\|\cdot\|$. If $\Delta^{k}$ is the standard $k$-simplex and $A_{k}$ is the space of $k$-simplices of a simplicial space $A$, then $|A|$ can be obtained as the quotient of the disjoint union of $\Delta^{k} \times A_{k}$ by first applying the equivalence relations $\left(x, 0^{*} a\right)=$ $\left(\theta_{*} x, a\right)$, using the face maps $\theta^{*}$ of $A$, and then applying the relation using the degeneracy maps $\theta_{*}$ of $A,\left(x, \theta_{*} a\right)=\left(\theta^{*} x, a\right)$. The space constructed after the first stage is $\|A\|$. For more details of the relationship between $\|\cdot\|$ and $|\cdot|$ see [7].

Let $s$ and $t$ be the source and target maps of $C$. For a topological space $X, S^{p} X$ will be the singular $p$-simplices on $X$, and $S X$ the total singular complex of $X$. For each $p \geqslant 0$ consider $S^{p} \bigodot$. We may view $S^{p} \bigodot$ as a discrete category with objects $S^{p} \vartheta$.

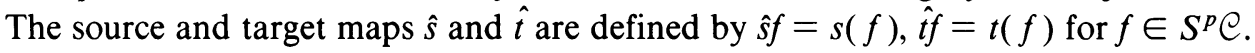
If $f, g \in S^{p} \bigodot$ their composition $f g$ is given by $f g(x)=f(x) g(x)$ for $x$ a point in the standard $p$-simplex.

Let $\mathcal{G}_{p} \mathcal{C}=\mathcal{G}_{0}\left(S^{p} \mathcal{C}\right)$ be the universal group of the discrete category $S^{p} \mathcal{C}$. Consider $\mathcal{G}_{*} \mathrm{e}=\cup_{p \geqslant 0} \mathcal{G}_{p} \mathrm{e}$. We have well-defined face maps $\partial_{i}: \mathcal{G}_{p} \mathcal{C} \rightarrow \mathcal{G}_{p-1} \varrho$ and well-defined

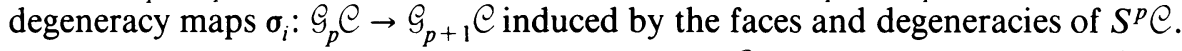

DEFINITION. The universal simplicial group of $\mathcal{C}$ is the simplicial group $\cup_{p \geqslant 0} \mathcal{G}_{p} \mathcal{C}$ together with the homomorphisms $\left\{\partial_{i}\right\}$ and $\left\{\sigma_{i}\right\}$.

We are interested in topological categories $C$ which have the property that the discrete categories $S^{p} C$ have fractions for each $p \geqslant 0$.

Definition. A topological category $C$ is said to have simplicial fractions if each of the discrete categories $S^{p} \bigodot, p \geqslant 0$, has fractions.

Note. A topological groupoid always has simplicial fractions. 


\section{Loops on the classifying space of a topological category.}

THEOREM 2. Let $巳$ be a topological groupoid which has simplicial fractions. Suppose

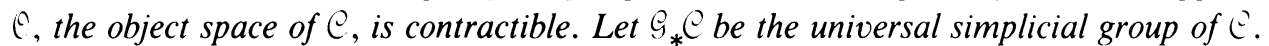
Then there is a weak homotopy equivalence $\left|\mathcal{G}_{*} \mathrm{e}\right| \rightarrow \Omega B \mathcal{C}$.

Theorem 2 is proved in exactly the same manner as the main theorem of [2] except that we use Theorem B of $\S 2$ (proved below) instead of Proposition 1.5 of [2].

For the proof of Theorem B we need only consider discrete categories with fractions, so for the remainder of this section $\mathcal{C}$ is discrete.

Proof of Theorem B. First consider the case in which $B E$ is connected. Choose $b \in \mathfrak{C}$, which will also represent a basepoint in $B \mathcal{C}$. Let $\widetilde{Y}$ be the free group on the points of $\theta$ modulo the one relation $b=1$. The following lemma describes $\mathcal{G}_{0} \mathcal{e}$ for an arbitrary discrete $\varrho$.

Lemma 1. There is an isomorphism $B: \mathcal{G}_{0} \mathcal{C} \rightarrow \pi_{1}(B \mathfrak{E}) * \widetilde{y} .($ Here $*$ denotes the free product of groups.)

This is proved for discrete groupoids in [3, pp. 16-17]. To adapt the proof to discrete categories we must replace the definition of basepath given there by the appropriate definition for discrete categories. Then the discussion and proof in [3] go over verbatim.

For $x \in \mathcal{e}$ let $x^{-1}$ denote the formal inverse of $x ; s\left(x^{-1}\right)=t(x)$ and $t\left(x^{-1}\right)=s(x)$. A basepath $\rho(q)$ from the basepoint $b \in \Theta$ to $q \in \Theta$ is a word $p(q)=x_{i}$ with $x_{i} \in \mathcal{E}$ satisfying $t\left(x_{i}^{ \pm 1}\right)=s\left(x_{i-1}^{ \pm 1}\right), s\left(x_{n}^{ \pm 1}\right)=b, t\left(x_{1}^{ \pm 1}\right)=q$.

Note an element of $\pi_{1}(B \mathcal{C})$ can be represented by a basepath with $s\left(x_{n}^{+1}\right)=$ $t\left(x_{n}^{ \pm 1}\right)=1$.

Choose once and for all a basepath $\rho(q)$ from $b$ to each object $q \in$ ?.

For $\alpha \in \mathcal{e}$ the word

$$
i(\alpha)=\rho(t(\alpha))^{-1} \cdot \alpha \cdot \rho(s(\alpha))
$$

represents an element of $\pi_{1}(B \mathcal{C})$. As in [3] the function $\Gamma \rightarrow \pi_{1}(B \mathcal{E}) * \widetilde{F}$, given by $\alpha \rightarrow t(\alpha)^{-1} * i(\alpha) * s(\alpha)$, induces the required isomorphism.

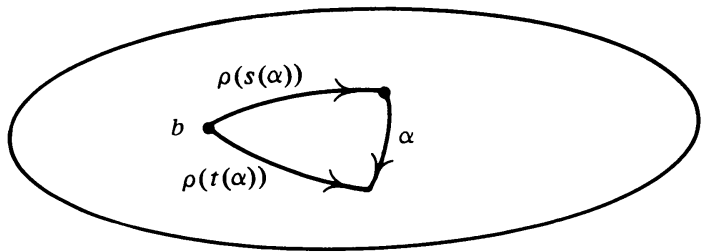

Note we are writing composition from right to left so our formulas are reversed from [3].

We continue to consider a category $\mathcal{E}$ with objects $\left[{ }^{\circ}\right.$ such that $B \mathcal{C}$ is connected. We construct a groupoid $\hat{e}$ with objects $\hat{\theta}$ as follows. Let the morphisms of $\hat{\mathcal{C}}$ be $\theta \times \theta \times \pi_{1}(B \bigodot)$ and $s(\alpha, \beta, x)=\alpha, t(\alpha, \beta, x)=\beta$. We have a simplicial map $N \mathcal{C} \rightarrow N \hat{\mathcal{C}}$ which is the identity on objects, and on morphisms is given by $f:$ $x \rightarrow(s(x), t(x),\langle x\rangle)$, where $\langle x\rangle$ is the generator of $\pi_{1}(B \mathcal{C})$ determined by $x \in \mathcal{C}$. 
${ }^{\top}$ passes to the quotient to give a diagram:

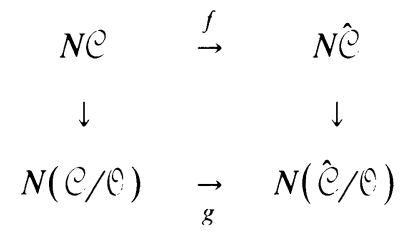

LEMMA 2. For $巳$ a discrete category,

(a) $|N \bigcup|$ is a $K\left(\pi_{1}(B \bigodot), 1\right)$,

(b) $|N \hat{e}|$ is a $K\left(\pi_{1}(B \bigodot), 1\right)$,

(c) $|N(\hat{\mathfrak{C}} / \mathfrak{C})|$ is a $K\left(\pi_{1}(B \mathcal{C}) * \mathscr{F}, 1\right)$.

Proof. For any category with fractions $\mathcal{C},|N \mathcal{Q}|$ is a $K(\pi, 1)[5]$ which implies (a) and (b) above (although for groupoids this fact is easy to prove directly). That $|N(\hat{\mathcal{e}} / \hat{C})|$ is a $K(\pi, 1)$ follows, because if $\hat{\mathcal{C}}$ is a groupoid then $\hat{\mathcal{e}} / \mathcal{C}$ has a pregroup structure $[4,9]$. In this case $\pi_{1}|N(\hat{\complement} / \mathcal{O})|=\mathscr{F} * \pi_{1}(B \circlearrowright)$ by Lemma 1 .

Now $\pi_{1}|N(\mathcal{C} / \Theta)|=\mathscr{F} * \pi_{1}(B C)=\mathcal{G}_{0}(\bigodot)$. We conclude the proof of the theorem (for $B \complement$ connected) by showing that $g$ induces a homotopy equivalence.

We have that $|N \mathcal{C}|$ and $|N \hat{C}|$ have the same homotopy type, so the same is true for the cones of the maps $|N \hat{\Theta}| \rightarrow|N \mathcal{C}|$ and $|N \hat{\Theta}| \rightarrow|N \hat{\mathcal{C}}|$. Equivalently the spaces $|N(\mathcal{C} / \mathcal{C})|$ and $|N(\hat{\mathrm{C}} / \mathcal{O})|$ have the same homotopy type.

The above proof applies equally well when $B C$ is not connected. In this case choose a basepoint $b_{\alpha} \in \mathcal{O}$ in each component. Then $\mathcal{G}_{0} \mathcal{C}=\mathscr{F}^{\prime}{ }_{{ }_{\alpha}} \pi_{1}\left(B \mathcal{C}, b_{\alpha}\right)$ where $\widetilde{F}^{\prime}$ is the free group on $\theta$ modulo the relations $b_{\alpha}=1$. Otherwise all of the details of the above proof go through without additional modifications.

\section{Proof of Theorem 1.}

Proof. Given $\Gamma$ and $\mathfrak{R}(\Gamma)$ as in the theorem, construct a topological category $\bigodot_{\Re(}(\Gamma)$ with objects $\left(\bigodot_{\Re}(\Gamma)\right)=\theta$ and morphisms $\left(\bigodot_{\Re}(\Gamma)\right)=\theta \times \Re(\Gamma)$. Denote by $s$ and $t$ the source and target maps of $\bigodot_{\Re}(\Gamma)$ given by $s(\alpha, f)=\alpha, t(\alpha, f)=t f(\alpha)$. We write $e(\Gamma)$ for $\bigodot_{\Re}(\Gamma)$ in the sequel, but this category depends on $\Re$ and $\Gamma$.

Since $\theta$ is contractible the continuous functor $e(\Gamma) \rightarrow \Re(\Gamma)$ defined by identifying $\Theta$ to the identity of $\Re(\Gamma)$ induces a homotopy equivalence $B C(\Gamma) \rightarrow B \mathscr{N}(\Gamma)$.

There is also a natural functor $F: C(\Gamma) \rightarrow \Gamma$ defined on morphisms by $(\alpha, f) \rightarrow$ $f(\alpha)$. We claim that this functor induces a weak homotopy equivalence $B C(\Gamma) \rightarrow B \Gamma$ which would prove the theorem.

Consider first $e(\Gamma)$ and $\Gamma$ with the discrete topology and their respective universal groups $\mathcal{G}_{0} \mathcal{C}(\Gamma)$ and $\mathcal{G}_{0} \Gamma$.

LEMmA 3. The functor $F: \mathcal{C}(\Gamma) \rightarrow \Gamma$ induces an isomorphism $F_{*}: \mathcal{G}_{0} \mathcal{C}(\Gamma) \rightarrow \mathcal{G}_{0} \Gamma$.

Proof. $F_{*}$ is onto: Let $x$ be a generator of $\mathcal{G}_{0} \Gamma$, that is, $x \in \Gamma$. Represent $x$ by a local section $\tilde{x}: U \rightarrow \Gamma$ such that $U \rightarrow t \tilde{x}(U)$ is a local homeomorphism. By our hypothesis there is an $m \in \Re(\Gamma)$ such that $\tilde{x} m$ is defined and in $\Re(\Gamma)$. Consider the element in $\mathcal{G}_{0} \mathcal{C}(\Gamma)$ represented by the word in the free group on $\Theta \times \mathscr{R}(\Gamma)$ given by $w=(\alpha, \tilde{x} m) \cdot(\alpha, m)^{-1}$ where $\operatorname{tm}(\alpha)=s(x)$. Then $F_{*} w=x$. 
$F_{*}$ is 1-1. Let $w=\left(\alpha_{1}, m_{1}\right)^{11} \cdots\left(\alpha_{n}, m_{n}\right)^{* 1}$ be an element of $\mathcal{G}_{0} \mathcal{Q}(\Gamma)$ such that $\bar{w}=m_{1}^{+1}\left(\alpha_{1}\right) \cdots m_{n}^{ \pm 1}\left(\alpha_{n}\right)=1$ in $\mathcal{G}_{0}(\Gamma)$. Without loss of generality we may assume $\bar{w}$ is itself a composable sequence of elements, that is, $t\left(m_{j}^{+1}\left(\alpha_{j}\right)\right)=s\left(m_{j-1}^{ \pm 1}\left(\alpha_{j-1}\right)\right)$ for $\mathfrak{Q}_{0} \Gamma$ is a pregroup, so it satisfies the regularity conditions of [4].

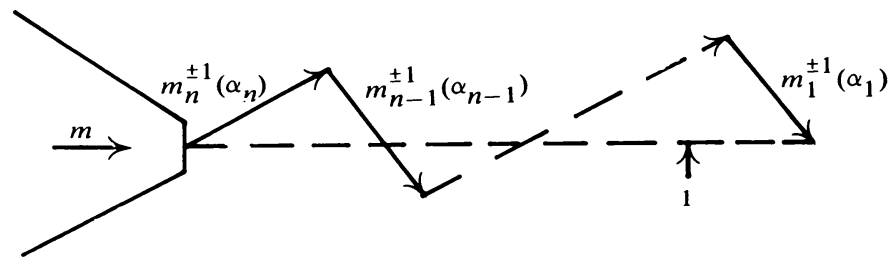

Then by $\Gamma$-completeness there exists an $m \in \mathfrak{M}(\Gamma)$ such that

$$
w \cdot(\alpha, m)=\left(\alpha_{1}, m_{1}\right)^{ \pm 1} \cdots\left(\alpha_{n}, m_{n}\right)^{ \pm 1}(\alpha, m)=(\alpha, m),
$$

where $\alpha$ is defined so that $m(\alpha)=\alpha_{n}$ and $m(\theta)$ is a sufficiently small neighborhood of $\alpha$. Therefore $w=1$ in $\mathcal{G}_{0} \mathcal{Q}(\Gamma)$ and $F_{*}$ is $1-1$. Note for this calculation, if $m(\theta) \subset n(\theta)$ and $m(\alpha)=n(\beta)$ then $(\beta, n)^{-1} \cdot(\alpha, m)=\left(\alpha, n^{-1} m\right)$ is in $\mathcal{G}_{0} \varrho(\Gamma)$ since $n^{-1} m \in \mathcal{M}(\Gamma)$.

Consider the open cover of $\theta$ by $\mathscr{U}=\{t f(\theta) \mid f \in \mathscr{M}(\Gamma)\}$. There is an induced open cover of $\Gamma$ by open sets of the form $\{x \mid s(x) \in U, t(x) \in V, U, V \in \mathcal{U}\}$. We also have an induced open cover on $N_{q} \mathcal{C}(\Gamma)$ for each $q \geqslant 0$. Consider the bisimplicial sets $\hat{S} N \mathcal{C}(\Gamma)=\cup_{p, q \geqslant 0} \hat{S}^{p} N_{q} \mathcal{C}(\Gamma)$ and $\hat{S} N \Gamma=\cup_{p, q \geqslant 0} \hat{S}_{p} N_{q} \Gamma$ consisting of singular simplices subordinate to the covers just given. It is not hard to see that $|\hat{S} N \mathcal{C}(\Gamma)| \rightarrow$ $B \mathcal{C}(\Gamma)$ and $|\hat{S} N \Gamma| \rightarrow B \Gamma$ are weak equivalences. Moreover, we have a well-defined bisimplicial map $F: \hat{S} N \mathcal{C}(\Gamma) \rightarrow \hat{S} N \Gamma$.

We wish to compare the vertical complexes of those bisimplicial sets.

Consider the discrete categories $\hat{S}^{p} \mathcal{C}(\Gamma)$ and $\hat{S}^{p} \Gamma$ and their universal groups $\mathcal{G}_{0}\left(\hat{S}^{p} \mathcal{C}(\Gamma)\right)$ and $\mathcal{G}_{0}\left(\hat{S}^{p} \Gamma\right)$. The proof of Lemma 3 applies in the same manner to the discrete categories $\hat{S}^{p} \mathcal{C}(\Gamma)$ and $\hat{S}^{p} \Gamma$ to show

LEMMA 4. The bisimplicial map $F: \hat{S} N \mathcal{C}(\Gamma) \rightarrow \hat{S} N \Gamma$ induces an isomorphism $F_{*}$ : $\mathfrak{G}_{0} \hat{S}^{p} \mathcal{C}(\Gamma) \rightarrow \mathcal{G}_{0} \hat{S}^{p} \Gamma$ for all $p \geqslant 0$, hence a simplicial isomorphism of the corresponding simplicial groups $\hat{\mathcal{G}}_{*} \mathrm{e}(\Gamma)=\cup_{p \geqslant 0} \mathcal{G}_{0} \hat{S}^{p} \mathrm{e}(\Gamma), \hat{\mathcal{G}}_{*} \Gamma=\cup_{p \geqslant 0} \mathcal{G}_{0} \hat{S}_{p} \Gamma$.

We verify now that $\mathcal{C}(\Gamma)$ has right simplicial fractions. The proof that $S^{p} \bigodot(\Gamma)$ has fractions for $p \geqslant 1$ is completely analogous to that for $p=0$. So for $p=0$ we consider a diagram $A \stackrel{x}{\rightarrow} B \stackrel{y}{\leftarrow} C$. That is, $x=(A, m), A \in \mathcal{C}, m \in \mathcal{M}(\Gamma)$ and $y=(C, n), C \in \mathcal{O}, n \in \mathcal{M}(\Gamma)$, and $B=n(C)=m(A)$. Let $\tilde{n}=\left.n\right|_{U}$ for $U$ an open set in $\theta$ such that $U \rightarrow \operatorname{tn}(U)$ is local homeomorphism and $\operatorname{tn}(U) \subset m(\theta)$. By $\Gamma$-completeness there is a $p \in \mathscr{M}(\Gamma)$ such that $\tilde{n}^{-1} m p$ is in $\mathscr{N}(\Gamma)$ with $\bar{B} \in \mathcal{O}$ such 
that $p(\bar{B})=A$. Set $\bar{x}=(\bar{B}, p), \bar{y}=\left(\bar{B}, \tilde{n}^{-1} m p\right)$. Then the following diagram verifies condition (1) for fractions:

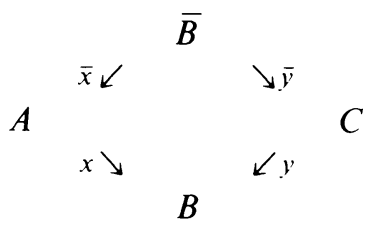

For condition (2) consider $x=(A, m), y=(A, n)$ with $m(A)=n(A)=B$. If there is a $z \in \mathcal{C}(\Gamma)$ so that $z x=x y$, then clearly there is an open set $U$ containing $A \in \Theta$ such that the local homeomorphisms $U \rightarrow \operatorname{tm}(U)$ and $U \rightarrow \operatorname{tn}(U)$ are identical. So by $\Gamma$-completeness there is a $p \in \mathfrak{N}(\Gamma)$ such that $m p=n p$. Choose $C$ so that $p(C)=A$. Then $w=(C, p)$ satisfies $x w=y w$.

We have proved that $S^{p} C(\Gamma)$ has right simplicial fractions. Then Theorem 2 applies to the following diagram to show that $A$ and $B$ are weak homotopy equivalences:

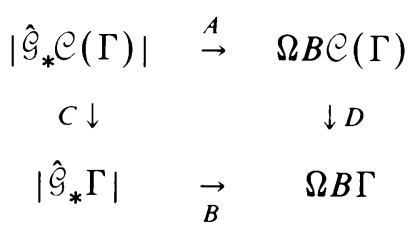

By Lemma 2, $C$ is a weak equivalence. Since we already have that $B C(\Gamma) \rightarrow B \Re(\Gamma)$ is a weak equivalence, the theorem is proved.

REMARK. The monoid $\mathfrak{M}(\Gamma)$ fails to satisfy property (1) for fractions: Consider $f, g \in \Re(\Gamma)$ so that $f(\theta) \cap g(\theta)=\varnothing$. There is no appropriate diagram associated to $\cdot \underset{f}{\vec{f}} \stackrel{\leftarrow}{\leftarrow} \cdot$.

6. Examples. (1) Let $\theta=\mathbf{R}^{n}$ and let $\Gamma=\Gamma_{n}^{r}$ be the groupoid of germs of local $C^{r}$ differentiable homeomorphisms of $\mathbf{R}^{n}, 1 \leqslant r \leqslant \infty$, with the sheaf topology. Then $\delta(\Gamma)$ is the monoid of $C^{r}$ immersions of $\mathbf{R}^{n}$ into itself. The main theorem gives a weak equivalence of $B \Gamma$ and $B$ ๆ $(\Gamma)$.

(2) Let $\theta$ and $\Gamma$ be as in Example (1) and let $\Re(\Gamma)=E(\Gamma)$ be the monoid of embeddings of $\mathbf{R}^{n}$ into itself. The proof of the main theorem applies since $E(\Gamma)$ is $\Gamma$-complete to give a weak equivalence of $B \Gamma$ and $B E(\Gamma)$. This equivalence is $\mathrm{G}$. Segal's theorem [8].

(3) Let $\theta=\mathbf{R}^{n}$ and $\Gamma=\Gamma_{n}^{\omega}$ be the sheaf of germs of local real analytic homeomorphisms of $\mathbf{R}^{n}$. The sections of the source map have arbitrarily small targets so they satisfy the hypothesis of the main theorem. We obtain a weak equivalence of $B \Gamma$ and $B S(\Gamma)$ and also $B \Gamma$ and $B E(\Gamma)$ in this case as well.

(4) The sheaf of germs of local holomorphic homeomorphisms.

(5) Let $\mathcal{\theta}=\mathbf{R}^{n}$ and let $\Gamma=V \Gamma_{n}^{r}, 1 \leqslant r \leqslant \infty, \omega$ be the sheaf of germs of volume preserving $C^{r}$ homeomorphisms of $\mathbf{R}^{n}$. P. Greenberg has observed that the hypotheses of the main theorem are satisfied so that we obtain a weak equivalence of $B \Gamma$ 
and $B \subseteq(\Gamma)$ in this case. On the other hand there are no arbitrarily small volume preserving embeddings of $\mathbf{R}^{n}$ into itself, so our theorem fails to give an equivalence of $B \Gamma$ and $B E(\Gamma)$ in this case. However, D. McDuff has proved that there is an equivalence if $n \geqslant 3$. Whether there is such an equivalence for $n=2$ is an open question.

\section{REFERENCES}

1. P. Greenberg, A model for groupoids of homomorphisms, Thesis, M. I. T., 1982.

2. S. Jekel, Loops on the classifying space for foliations, Amer. J. Math. 102 (1980), 13-23.

3. ___ Simplicial decomposition of $\Gamma$-structures, Bol. Soc. Mat. Mexican 26 (1981), 13-20.

4. Simplicial K( $G, 1)$ 's, Manuscripts Math. 21 (1977), 189-203.

5. D. Kan and W. Dwyer, Calculating simplicial localizations, J. Pure Appl. Algebra 18 (1980), 1-16.

6. D. Quillen, Spectral sequences of a double semi-simplicial group, Topology 5 (1966), 155-157.

7. G. Segal, Categories and cohomologv theories, Topology 13 (1974), 293-312.

8. Classifying spaces related to foliations, Topology 17 (1978), 367-382.

9. J. Stallings, The cohomology of pregroups, Lecture Notes in Math., vol. 319. Springer-Verlag. New York, 1973, pp. 169-182.

Department of Mathematics, Northeastern University, Boston, Massachusetts 02115 